\title{
Enteritis lúpica. Reporte de un caso
}

\author{
Alvaro Herrera Escandon ${ }^{1}$, Juan Manuel Molina Gomez², Jorge Manuel Rueda Gutierrez ${ }^{3}$, Herman Gonzalez \\ Buritica $^{4}$ \\ ${ }^{1}$ Médico Residente III Medicina Interna, Universidad Libre Seccional Cali. \\ ${ }^{2}$ Médico Interno, Universidad Libre Seccional Cali. \\ ${ }^{3}$ Médico Internista Reumatólogo, Profesor Asistente Universidad Libre Seccional Cali, Centro Médico Imbanaco Cali - Colombia. \\ ${ }^{4}$ Médico Internista Reumatólogo, Profesor Asistente Universidad Libre Seccional Cali, Centro Médico Imbanaco Cali - Colombia.
}

\section{Resumen}

Las manifestaciones en lupus son variadas y algunas de ella con menor frecuencia. Presentamos el caso de una paciente con diarrea crónica en el contexto de un lupus eritematoso sistémico. Luego de su estudio, se evidenciaron hallazgos clínicos y radiológicos compatibles con actividad lúpica que sugirieron la posibilidad diagnóstica de enteritis lúpica. Se indicó tratamiento inmunosupresor y al cabo de 30 días se logró la resolución completa de la sintomatología. En la actualidad se desconoce la terapia inmunosupresora más acertada para esta manifestación de la enfermedad, siendo el uso de corticoides la principal herramienta terapéutica.

\section{Abstract}

The clinical findings of lupus are diverse and some of them exotic. Herein, we present the case of a patient with chronic diarrhea and lupus. We found clinical and radiologic features that suggested lupus activity. Complete resolution of symptoms was achieved after immunosuppressive therapy; however, more evidence is needed regarding the appropriate therapeutic approach to cases like this.

\section{Introducción}

A partir de su primera descripción en el siglo XIX fueron necesarios más de 100 años para determinar que el Lupus Eritematoso correspondía a una enfermedad sistémica $(\mathrm{LES})^{1}$. La prevalencia de esta entidad está estimada entre 7.4 a 159.4 casos por 100.000 habitantes en el mundo, la relación mujer: hombre es 9:1, con pico de incidencia entre 15 a 40 años ${ }^{2,3}$.

El LES es una enfermedad multifactorial y de espectro multisistémico; sus manifestaciones más frecuentes son: las lesiones mucocutáneas, el compromiso músculo esquelético, las serositis, el aspecto neuropsiquiátrico y las citopenias ${ }^{4}$. Las manifestaciones gastrointestinales pueden simular otras condiciones como infecciones, y aunque su prevalencia es alta (25-40\%) el compromiso de la enfermedad allí es raro y éstas pueden ser difíciles de evidenciar ${ }^{5}$.

La enteritis lúpica es una de las formas de manifestación gastrointestinal del compromiso por LES. Ésta cursa con dolor abdominal, náuseas, vómitos y diarrea. El inicio rápido de estos síntomas obliga a determinar condiciones amenazantes de la vida, pero su detección se convierte en un desafío para el clínico. Se presenta el caso de una paciente con historia de LES de 10 años de evolución con dolor abdominal, diarrea y ascitis ${ }^{6}$.

\section{Descripción del caso}

Paciente femenina de 59 años con cuadro clínico de 6 meses de diarrea intermitente, hematoquexia intermitente, dolor abdominal difuso tipo cólico, vómitos y pérdida de peso del $12 \%$ del peso inicial ( $50 \mathrm{~kg}$ ). Los diez días previos a su consulta reaparece la diarrea, de manera frecuente, voluminosa, acompañada de vómitos y dolor abdominal tipo cólico por lo que acude a urgencias. Tiene antecedente patológico de lupus eritematoso sistémico desde 2003, padeció de artralgias, rash malar, anticuerpos antinucleares $1 / 2560$ durante el desarrollo de los síntomas, anti DNA 1/320, Anti Ro: 23 U/L, RNP 27 U/L (valor normal $<20 \mathrm{U} / \mathrm{L})$. Además, hipotiroidismo y osteoartrosis. Recibe tratamiento con Cloroquina desde 2003, 250 mg/día, y desde 2011 Hidroxicloroquina $200 \mathrm{mg} / \mathrm{día}$, Levotiroxina $50 \mathrm{mcg} /$ día y sulfato de glucosamina intermitente. En remisión del LES por 2 años.

\section{Correspondencia}


Al examen físico presentaba TA 110/70 mmHg, FC 84 lat/min, FR 18 respiraciones/min, signos de emaciación, palidez conjuntival, a nivel cardiopulmonar normal, dolor abdominal superficial en hemiabdomen superior sin visceromegalias, onda ascítica positiva, no irritación peritoneal, piel seca con signos de pliegue presentes. Se toman exámenes iniciales (Tabla 1).

Se reanimó con líquidos endovenosos, se repuso fósforo y pauta antibiótica para diarrea bacteriana con ceftriaxone + metronidazol por 7 días. Por hallazgos clínicos iniciales, se ordenó ultrasonografía de abdomen para determinar la presencia de ascitis. Ésta reportó líquido libre en cavidad como único hallazgo por lo que se realiza paracentesis diagnóstica.

El líquido ascítico (Tabla 2) indicaba exudado de tipo inflamatorio con células atípicas, el gradiente de líquido ascítico $<1,1$, el cultivo negativo para bacterias y hongos, test de auramina negativo, reacción en cadena de polimerasa (PCR) para tuberculosis en líquido ascítico negativa y cultivo para micobacterias final negativo. Por persistencia de los síntomas se ordena tomografía de abdomen contrastada y endoscopia digestiva. La primera reporta engrosamiento marcado del antro gástrico, duodeno y yeyuno proximal, líquido libre en calidad, signo de tiro al blanco en asas intestinales, engrosamiento de asas de intestino delgado así como congestión vascular mesentérica y signo del peine (Figuras 1 y 2). En la endoscopia digestiva se observó engrosamiento de la mucosa pre pilórica y duodenal con imagen hacia la luz que producía efecto de masa, las biopsias informaron la presencia de infiltrado linfoplasmocitario con polimorfonucleares neutrófilos sin evidencia de atipias celulares, la citología de líquido ascítico reportaba infiltrado mononuclear sin evidencia de células neoplásicas. En vista de todos estos hallazgos, y al no tener una etiología clara, se lleva a cabo laparoscopia diagnóstica donde no se encuentran evidencias de siembras metastásicas o lesiones peritoneales.

Durante la evolución clínica mostró persistentemente dolor abdominal, diarrea intermitente y vómitos sin tolerancia a la vía oral; requirió alimentación enteral por sonda. Presenta episodio de descenso marcado de la hemoglobina sin sangrado digestivo clínico, con prueba de coombs directo positiva, se toman marcadores de actividad lúpica reportados como positivos y consumo del complemento (Tabla 1). Se interpreta que cursa con actividad de la enfermedad de base (LES) y su manifestación clínica correspondiente a una enteritis lúpica (hallazgos clínicos y radiológicos). Se indican pulsos de metilprednisolona a dosis de $500 \mathrm{mg} /$ día por 3 días y con posterior paso de medicación a corticoide a $1 \mathrm{mg} / \mathrm{kg} /$ día. Presenta franca mejoría clínica, tolerancia a la vía oral y retiro de su dispositivo de alimentación en la primera semana, recuperación de peso, apetito y resolución de la ascitis en los primeros 30 días después del inicio del tratamiento.

\section{Discusión}

El Lupus Eritematoso Sistémico (LES) presenta comorbilidades como infecciones, ateroesclerosis y malignidades que son potencialmente fatales y empeoran el curso de la enfermedad.

Las manifestaciones gastrointestinales en LES son variadas; de éstas, cerca del $40 \%$ de los pacientes acusan dolor abdominal agudo, úlceras orales (7-52\%), anormalidad en las pruebas hepáticas (>60\%), disfagia (1-13\%) y hepatitis autoinmune $(2-5 \%)^{7}$. Su abordaje diagnóstico exi-

\begin{tabular}{|l|c|}
\hline LEUCOCITOS & $6.290 \mathrm{cel} / \mathrm{mm}^{3}$ \\
\hline LINFOCITOS & $420 \mathrm{cel} / \mathrm{mm}^{3}(6,7 \%)$ \\
\hline NEUTRÓFILOS & $5.820 \mathrm{cel} / \mathrm{mm}^{3}(92 \%)$ \\
\hline EOSINÓFILOS & $40 \mathrm{cel} / \mathrm{mm}^{3}(0 \%)$ \\
\hline HEMOGLOBINA & $9,6 \mathrm{~g} / \mathrm{dl}$ \\
\hline HEMOGLOBINA CORPUSCULAR MEDIA & 31 \\
\hline VOLUMEN CORPUSCULAR MEDIO & $86 \mathrm{fL}$ \\
\hline HEMATOCRITO & $29,50 \%$ \\
\hline PLAQUETAS & $218 X 10 \wedge 3 / \mathrm{UL}$ \\
\hline AMILASA EN SUERO & $85 \mathrm{U} / \mathrm{L}$ \\
\hline CREATININA & $0,64 \mathrm{mg} / \mathrm{dl}$ \\
\hline SODIO & 140 \\
\hline POTASIO & 4,0 \\
\hline FÓSFORO & $1,84(2,4-5,0)$ \\
\hline CALCIO IÓNICO & $1,04 \mathrm{mmol} / \mathrm{L}$ \\
\hline TEST DE BAERMAN & Negativo \\
\hline ANTICUERPOS ANTI DNA & $1 / 40 \mathrm{patrón}$ \\
\hline ANTICUERPOS ANTI RO & $27 \mathrm{U} / \mathrm{L}$ \\
\hline ANTICUERPOS ANTI LA & $<10 \mathrm{U} / \mathrm{L}$ \\
\hline ANTICUERPOS ANTI RNP & $37 \mathrm{U} / \mathrm{L}$ \\
\hline ANTICUERPOS ANTI SM & $<10 \mathrm{U} / \mathrm{L}$ \\
\hline ANTICUERPOS ANTINUCLEARES & $1 / 1280 \mathrm{patrón} \mathrm{homogéneo}$ \\
\hline COOMB DIRECTO & ++ \\
\hline ALBÚMINA & $0,5 \mathrm{~g} / \mathrm{L}(>0,9)$ \\
\hline COMPLEMENTO C3 & $0,005 \mathrm{~g} / \mathrm{L}(>0,1)$ \\
\hline COMPLEMENTO C4 & $184 \mathrm{U} / \mathrm{dl}(120-246)$ \\
\hline DESHIDROGENASA LÁCTICA & $49 \mathrm{~mm} / \mathrm{h}$ \\
\hline VELOCIDAD DE ERITROSEDIMENTACIÓN & $21 \mathrm{mg} / \mathrm{dl}$ \\
\hline Proteina C reactiva & hay esquistocitos \\
\hline EXTENDIDO DE SANGRE PERIFÉRICA & \\
\hline
\end{tabular}

Tabla 1. Resultado de exámenes. 


\begin{tabular}{|l|}
\hline LíQUIDO ASCíTICO \\
\hline Eritrocitos $17 \times \mathrm{mm}^{3}$ \\
\hline Leucocitos $67 \mathrm{~mm}^{3}$ \\
\hline Neutrófilos $73 \%$ \\
\hline Ligeramente turbio \\
\hline Amarillo \\
\hline Glucosa $119 \mathrm{mg} / \mathrm{dl}$ \\
\hline Proteínas $3 \mathrm{~g} / \mathrm{dl}$ \\
\hline Albúmina $2,0 \mathrm{~g} / \mathrm{dl}$ \\
\hline Amilasa $30 \mathrm{U} / \mathrm{L}$ \\
\hline Fosfatasa alcalina $11 \mathrm{U} / \mathrm{L}$ \\
Albúmina sérica $3,0 \mathrm{GASA} 1,0$ \\
\hline LDH $345 \mathrm{U} / \mathrm{L}$ (<215) \\
\hline
\end{tabular}

Tabla 2. Resultado citoquímico de líquido ascítico.

ge descartar causas medicamentosas de dolor abdominal y compromiso hepático, obliga a establecer la presencia de infecciones, fenómenos trombóticos vasculares gastrointestinales, complicaciones de disfunción orgánica y otras enfermedades. Estudios retrospectivos han mostrado que el dolor abdominal agudo en LES que se lleva a exploración quirúrgica en el $73 \%$ de los casos se documentaba vasculitis intestinal y de estos cerca del 50\% fallecían, lo que permite inferir que esta manifestación es ominosa y de alta mortalidad ${ }^{8,9}$.

En otros reportes de la literatura se sugiere que, una vez considerado el dolor abdominal relacionado con la enfermedad, existe un espectro de entidades a descartar que expliquen los síntomas de los pacientes con LES y manifestaciones gastrointestinales (vasculitis mesentérica, colecistitis acalculosa, enfermedad ulceropéptica y pancreatitis). En estas revisiones se ha determinado que la incidencia de enteritis lúpica es baja $(0,2 \%)$, pero en otras series reportan frecuencias del $53 \%$ de las consultas con dolor abdominal agudo en $\mathrm{LES}^{10}$.

La Enteritis Lúpica como entidad aislada fue descripta por Hoffman y Katz en $1980^{16}$, donde fue considerada una manifestación rara del LES. Su principal compromiso es observado en yeyuno e íleon con escasa afectación en recto por la circulación colateral y llamativamente sin descripciones en duodeno ${ }^{11}$.

Descripta como tal en BILAG 2004 como "vasculitis o inflamación del intestino delgado, con imagen radiográfica de soporte y/o biopsia la cual subraya la amplitud del espectro de la enfermedad"12. En 2013 se reportaron 7 casos que consideraban un grupo de síntomas como principales de la enfermedad (dolor abdominal (97\%), ascitis $(78 \%)$, náusea (49\%), vómitos (42\%), diarrea (32\%), fiebre (20\%). Así mismo se observa la presencia de anti-

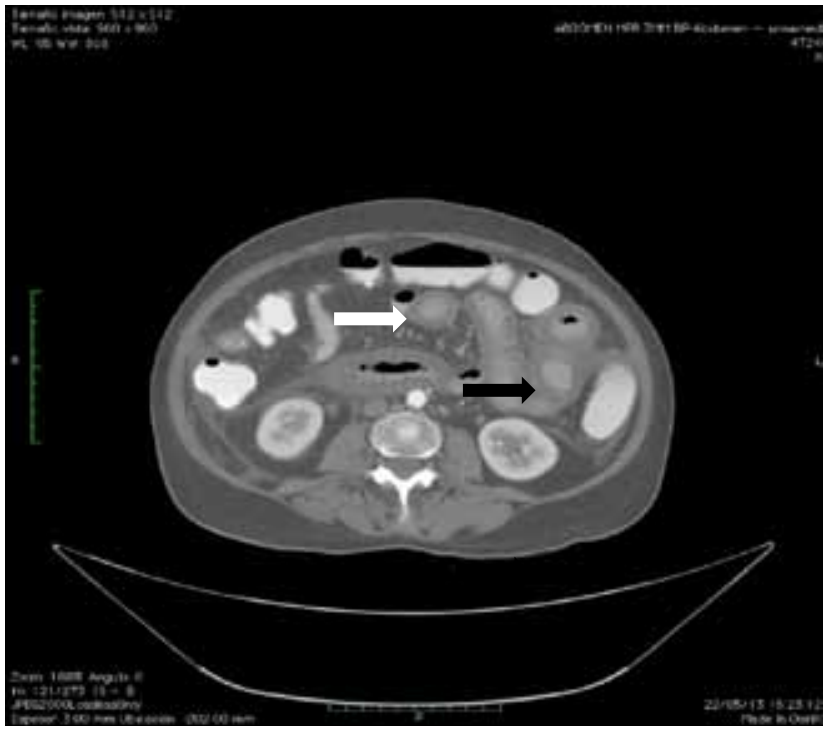

Figura 1. Tomografía contrastada de abdomen. En la flecha blanca se observa signo de tiro al blanco, presencia de doble halo y realce periférico al contraste. En la flecha negra se demuestra el marcado engrosamiento de la pared del intestino.

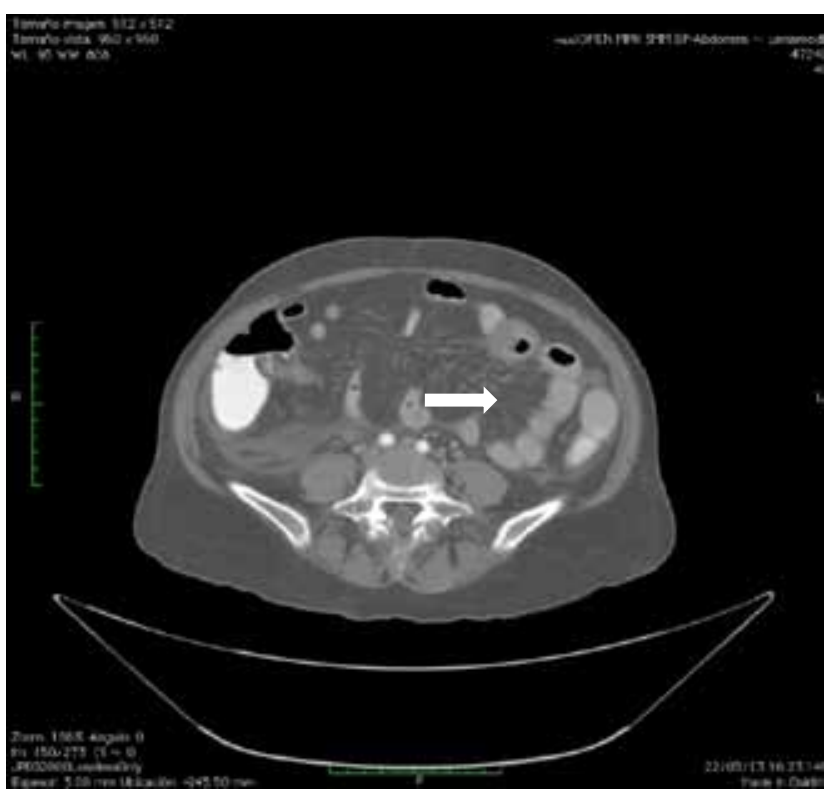

Figura 2. Tomografía contrastada de abdomen. Se observa congestión vascular mesentérica y signo del peine.

cuerpos antinucleares, anti DNA, anemia hemolítica e hipocomplementemia como características de esta serie de pacientes y compartidos con el caso de nuestra paciente $^{13}$. El perfil de autoanticuerpos no varía con respecto a los grupos de control en las otras manifestaciones de la enfermedad, siendo poco probable la determinación del intestino como órgano diana afectado ${ }^{14}$. Las manifestacio- 
nes radiológicas de signos de tiro al blanco, peine y edema de pared intestinal son claves al momento del diagnóstico y orientan al clínico para el inicio del tratamiento ${ }^{15}$.

El diagnóstico definitivo es realizado con base en las manifestaciones clínicas, radiológicas y/o la toma de biopsias con exclusión de otras causas de dolor abdominal, diarrea y ascitis. El alivio de los síntomas una vez instaurado el tratamiento es dramático y oscila entre dos días a ocho semanas para su respuesta, lo que se corrobora con la mejoría biológica y radiológica.

El tratamiento en la gran mayoría de reportes de la literatura es basado en esteroides a altas dosis para inducción de remisión, seguidos por dosis de mantenimiento del mismo con descenso gradual y acompañados de hidroxicloroquina, azatioprina o micofenolato mofetilo. La tasa de recaídas al año ocurre en un cuarto de los pacientes bajo tratamiento y por ahora no se conocen factores predictores de esta complicación ${ }^{14}$.

Algunos autores han planteado el tratamiento con rituximab en aquellos pacientes con múltiples recaídas a pesar de la terapia inmunosupresora y dosis altas de corticoides. En estos se ha demostrado la incapacidad para la reducción de la dosis de esteroides, de manera que existe reducción de los síntomas tras el inicio del rituximab, correlacionándose con el descenso del corticoide y la reducción de linfocitos B CD19 positivos ${ }^{17}$. En conclusión, las manifestaciones gastrointestinales en LES son frecuentes, requieren en primera instancia la exclusión de entidades potencialmente fatales, sin obviar posibilidad de la actividad de la enfermedad. El diagnóstico de enteritis lúpica requiere un cuadro clínico compatible, imágenes concordantes y/o biopsias de intestino delgado. La respuesta clínica a los corticoides es adecuada y hoy en día existen terapias alternativas para su recurrencia y falla terapéutica que se encuentran en investigación.

\section{Bibliografía}

1. Tsokos GC. Systemic lupus ery thematosus N Engl J Med 2011; 365:2110-21.

2. Danchenko N, Satia JA, Anthony MS. Epidemiology of systemic lupus erythematosus: A comparison of worldwide disease burden. Lupus 2006; 15:30818.

3. Masi AT, Kaslow RA. Sex effects in systemic lupus erythematosus: A clue to pathogenesis. Arthritis Rheum 1978; 21:480-4.

4. Dubois EL, Tuffanelli DL: Clinical manifestations of systemic lupus erythematosus: computer analysis of 520 cases, JAMA 1964; 190:104-111.
5. Schulz SW, Derk CT. The Gastrointestinal Manifestations of Systemic Lupus Erythematosus: A Survey of the Literature. The Open Autoimmunity Journal 2009; 1:10-26.

6. Harley JB, Alarcon-Riquelme ME, Criswell LA, et al: Genome-wide association scan in women with systemic lupus erythematosus identifies susceptibility variants in ITGAM, PXK, KIAA1542 and other loci, Nat Genet 2008; 40(2):204-210.

7. Vergara-Fernandez O, Zeron-Medina J, MendezProbst C, Salgado-Nesme N, Borja-Cacho D, Sanchez-Guerrero J, Medina-Franco H. Acute abdominal pain in patients with systemic lupus erythematosus. J Gastrointest Surg 2009; 13:1351-7.

8. M. Witt, D. Zecher, H.-J. Anders. Gastrointestinal manifestations associated with systemic lupus erythematosus Eur J Med Res 2006; 11:253-260.

9. Zizic TM, Classen JN, Stevens MB. Acute abdominal complications of systemic lupus erythematosus and polyarteritis nodosa. Am J Med. 1982; 73:52531.

10. Drenkard C et al. Vasculitis in systemic lupus erythematosus. Lupus 1997; 6:235-42.

11. Agmon-Levin N, Mosca M, Petri M, Shoenfeld Y: Systemic lupus erythematosus one disease or many? Autoimmun Rev 2012, 11(8):593-595.

12. Yee CS, Farewell V, Isenberg DA, Griffiths B, Teh LS, Bruce IN, The BILAG-2004 index is sensitive to change for assessment of SLE disease activity, Rheumatology 2009; 48:691-695.

13. Janssens P, Arnaud L, Galicier L, Mathian A, Hie $\mathrm{M}$, Sene $\mathrm{D}$ et al. Lupus enteritis: from clinical findings to therapeutic management. Orphanet Journal of Rare Diseases 2013; 8:67.

14. C-K Lee, M S Ahn, E Y Lee, J H Shin, Y-S Cho, H K Ha, B Yoo, H-B Moon. Acute abdominal pain in systemic lupus ery thematosus: focus on lupus enteritis (gastrointestinal vasculitis). Ann Rheum Dis 2002; 61:547-550.

15. Ha HK, Lee S, Rha SE, Kim JH, Byun JY, Lim HK et al. Radiologic Features of Vasculitis Involving the Gastrointestinal Tract. RadioGraphics 2000; 20:779-794.

16. Hoffman BI, Katz WA. The gastrointestinal manifestations of systemic lupus erythematosus: a review of the literature. Semin Arthritis Rheum. 1980 May; 9(4):237-247.

17. JS Oh, Y-G. Kim, SG Lee, C-K. Lee and B. Yoo. Successful treatment of recurrent lupus enteritis with rituximab, Lupus 2010; 19:220. 